\title{
Recognition of handwritten Arabic (Indian) numerals using skeleton matching
}

\author{
Bassam A. Y. Alqaralleh, Malek Z. Alksasbeh, Tamer Abukhalil, Harbi Almahafzah, \\ Tawfiq Al Rawashdeh \\ Faculty of Information Technology, Al Hussein Bin Talal University, Jordan
}

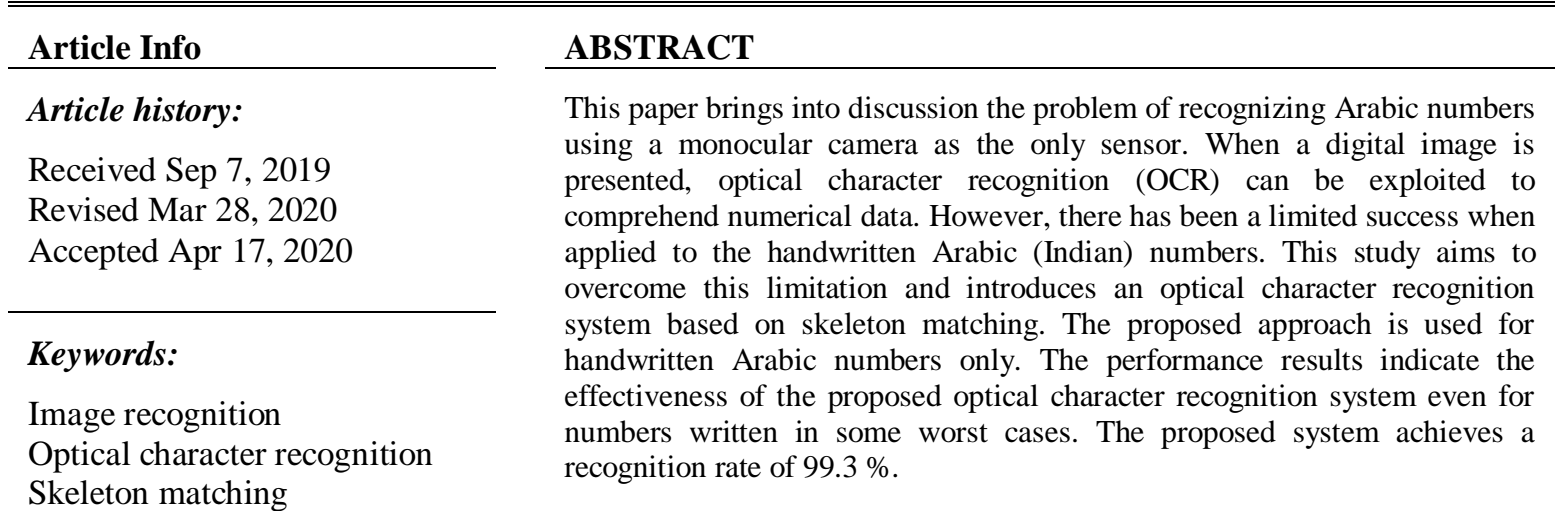

Copyright $\odot 2020$ Institute of Advanced Engineering and Science. All rights reserved.

\section{Corresponding Author:}

Malek Zakarya Alksasbeh, Faculty of Information Technology, Al Hussein Bin Talal University, King Hussien Bin Talal University Str، Ma'an, Jordan. Email: malksabeh@ahu.edu.jo

\section{INTRODUCTION}

Recognition of handwritten characters which is also known as optical character recognition (OCR) has been a significant problem in the field of digital image processing. OCR is the process of autonomous recognition and identification of characters extracted from digital images. When the image contains handwritten symbols, it becomes very vital to comprehend such writing especially in case of recognizing numerals. Numerals recognition is used in multiple applications such as identity authentication, bank check processing in auto teller machines (ATMs), data entry, etc. [1]. For such applications, the performance is measured in terms of accuracy and speed. Flawless computer recognition of these numbers and characters is the key factor when designing the system. The problem of recognizing handwritten digits is also considered as one of the most important problems facing pattern recognition, pattern classification and digital image processing especially for Arabic numbers. Due to the increasing usage of handwritten Arabic numbers in many businesses worldwide, it has become essential to computers to understand such numerals.

Many researchers have discussed and introduced computational intelligent techniques to accurately recognize handwritten Arabic text and numbers. Multiple OCR approaches have been introduced in the literature, including those based on Neural Networks (NN) [2, 3], fractal code [4], fuzzy-logic [5], profiles [6], SVM's [7, 8], and Hidden Markov Models (HMMs) [9]. Other methods have been considered novel for the area of recognizing characters using template matching such as in [10]. Other authors have investigated and proposed different solutions of OCR using feature extraction as in [8,9], windowing [11], centralized moments [12], and discrete wavelet transform [13, 14]. Al-Omari [15] for instance, used a template matching scheme after applying an average filter for Arabic numerals. He also discussed the use of feature vectors which represent a set of boundary distances from a pivot point called the center of gravity (COG) of the 
number. HMM technique is used by the authors in [16]. They proposed a technique that takes advantage of a sliding window method in the stage of feature extraction. The window is basically a region that changes its size and position to be able to move and adjust itself to the written line which contains the distributed Arabic words and characters.

Character recognition methods that use support vector machine (SVM) were also investigated by several authors. Takruri et al. [17] came up with an approach that uses multiple SVMs being run in parallel to classify characters and numerals. The results were then categorized into digits (0-9) and characters (A-Z). The two groups are then fed into another set of SVMs to individually classify each object. A recognition rate of $97.2 \%$ was achieved. However, because large numbers of SVMs were used in their system, the performance was significantly degraded in terms of speed. On the other hand, Mahmoud and Al-Khatib [18] used Gabor-based filters to extract features before feeding the image to a k-nearest neighbor (KNN) and SVM classifiers. They achieved the highest two average recognition rates: $99.95 \%$ and $98.75 \%$. However, those figures drop dramatically when noise is presented in the image.

Segmentation is an important stage when recognizing objects in a digital image. Fuzzy C-Means (FCM) algorithm [19] is one of the most powerful fuzzy clustering methods which have been used in image segmentation. FCM is considered to be robust to ambiguity and can obtain much extensive data about objects than regular segmentation methods [20]. An asymmetrical segmenting pattern recognition method to retain a feature vectors for the handwritten Arabic numbers was presented in [21]. The feature vectors were fed into a Neural Network classifier with a reported recognition rate of $97.6 \%$. Also, a novel three-level approach for classification of isolated handwritten Arabic numerals was introduced in [5]. After being cropped, resized and vectorized, the binary image vector of the handwritten numeral was fed to an FCM classifier stage followed by an SVM classifier stage to decrease the misclassification rate of the FCM.

Ghaleb et al. [22] calculated the occurrences of digits using horizontal and vertical weighted centroid moments and minimum distance classifier. The authors in [9] proposed a multiple feature/resolution scheme for recognizing Arabic numbers using Hidden Markov Models (HHMs). The proposed method uses scale and translation invariant affine transformations combined with HMM classifiers to recognize handwritten Arabic numbers. They proposed multi-resolution feature extraction approach that generates a set of GSC (gradient, structure, and concavity) features out of each character. The multi-resolution features extraction was very helpful in recognizing common patterns when numbers are handwritten such as edge curvature in a neighborhood of pixels, short strokes types over several pixels and certain concavities which may exist across the image. However, adding complexity to the recognition system often cause performance issues.

In order to cope with previous drawbacks, smaller vector sizes have been used for segmentation of an image. Ahmad and Maen [23] represented a structural online approach for the Arabic digits recognition. The shape-based features are extracted by vectoring the primary and secondary sub-patterns through identifying the changes in the slope sign, and then matching has been done using their special grammar which compares the string of primitives to the input digit. Das et al. [24] combined PCA/MPCA (Principal Component Analysis/Modular PCA) and QTLR (Quad-tree based hierarchically derived Longest-Run) for extracting the features of handwritten numerals of five popular scripts including Arabic. They used SVM classifier on database of CMATER db 3.4.1 which consists of 3000 samples. The KHATT database [25] is a comprehensive offline handwritten Arabic text database that is designed to be used in Arabic text recognition research, writer identification and verification research and Arabic forms analysis research.

However, as mentioned above, several researchers have introduced algorithms for Arabic and Persian numbers recognition. The real challenge in this context is successfully recognizing handwritten numbers as opposed to machine printed numbers. A typical OCR system consists of the following stages: acquisition, preprocessing, segmentation, feature extraction, and classification [26]. These phases are also the fundamental building blocks of machine vision system. Preprocessing stage involves converting the RGB image into gray scale digital one and some enhancements such as noise removal. In the segmentation phase, partitioning the monochrome image into its main components such as the related foreground objects and the background is accomplished. Segmentation also produces several labeled regions or sub-images. Arabic language is a cursive based language where letters in each word are connected and attached to each other in a continuous form. While both printed and handwritten Arabic text is a cursive one, Arabic (Indian) numerals are not cursive. Therefore, the process of recognizing numbers using computers is simplified. More specifically, the part where segmentation is required will be eliminated unlike the case with Arabic text.

Feature extraction phase converts the digital image into a set of numerical vectors using algorithms responsible for measuring the color (intensity), the texture and the shape of the segmented objects to assist in the classification process. Feature extraction also involves adjustment operations for truncation and normalization. It also includes operations such as eliminating the gaps that surround the objects inside the image using methods such as binarization, and skeleton generating. In our case, once the major features of the image and its relevant objects (features of numerals) are extracted and encoded into a feature vector, 
the skeleton matrices will be generated and will be fed into the pattern classification stage. At this point, image processing reaches its final phase which aims to identify each individual number via assigning a label to it. The set of produced labeled numbers will be further used in the original application. Table 1 presents the Arabic numbers which are also known as old Indian numbers. Also, Figure 1 presents the number "TWO" written in two different common Arabic writing styles.

Table 1. Arabic digits and its Equivalent English digits

\begin{tabular}{cc}
\hline Arabic Number & Corresponding English number \\
\hline 0 & 0 \\
1 & 1 \\
2 & 2 \\
3 & 3 \\
4 & 4 \\
5 & 5 \\
6 & 6 \\
7 & 7 \\
8 & 8 \\
9 & 9 \\
\hline
\end{tabular}

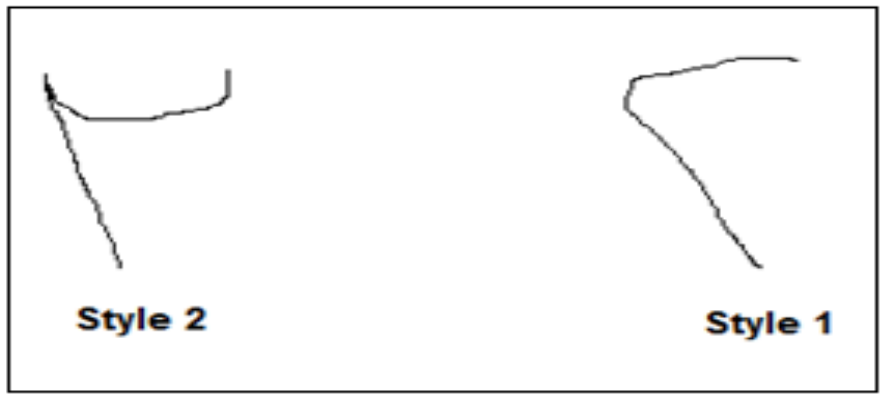

Figure 1. Number "TWO” in Arabic, written by Hand in Two different common writing styles

However, Arabic numbers have a great importance in regions where Arabic language is the official language. Arabic numbers are mainly used to express numeral values in any context in the Middle East and part of Asia. The wide use of such numbers encourages inventing computer-based systems that can read and recognize handwritten numbers or machine printed ones. In view of the fact that handwritten Arabic alphabets and numerals have no wide research yet. This study proposes an innovative technique for precise automated recognition of Arabic handwritten numerals. This technique adapted the OCR Skeleton matching method which was introduced by [27]. The proposed system achieves a recognition rate of $99.3 \%$. This approach involved text acquisition and filtering method, thinning algorithm design, segmentation technique, feature extraction and classification strategy. In addition, this study focus on recognizing the numbers that most likely appear in a multi-digit number format as shown in Figure 2.

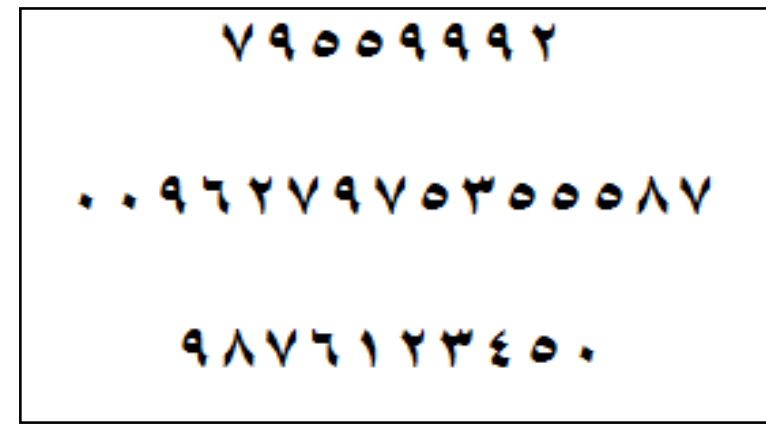

Figure 2. Examples of multi-digit Arabic number series 


\section{RESEARCH METHOD AND PROPOSED SYSTEM}

As mentioned earlier, the OCR System Skeleton matching introduced by [27] has been adapted in this study. The main stages of the Skeleton processes can be categorized as preprocessing, recognition, self-learning and post-processing. The main components of the system are shown in Figure 3. Skeletonization has been modified to include a three-steps approach. However, before the proposed approach is applied on the input image, a thinning process is executed on the image. The application of the thinning process uncovers the connected contours of each scanned object until it reaches the most medial one-pixel width. The efficiency of the thinning method is measured by the extent to which the skeleton has been extracted while preserving the overall topology of the objects' shape without many unavoidable imperfections.

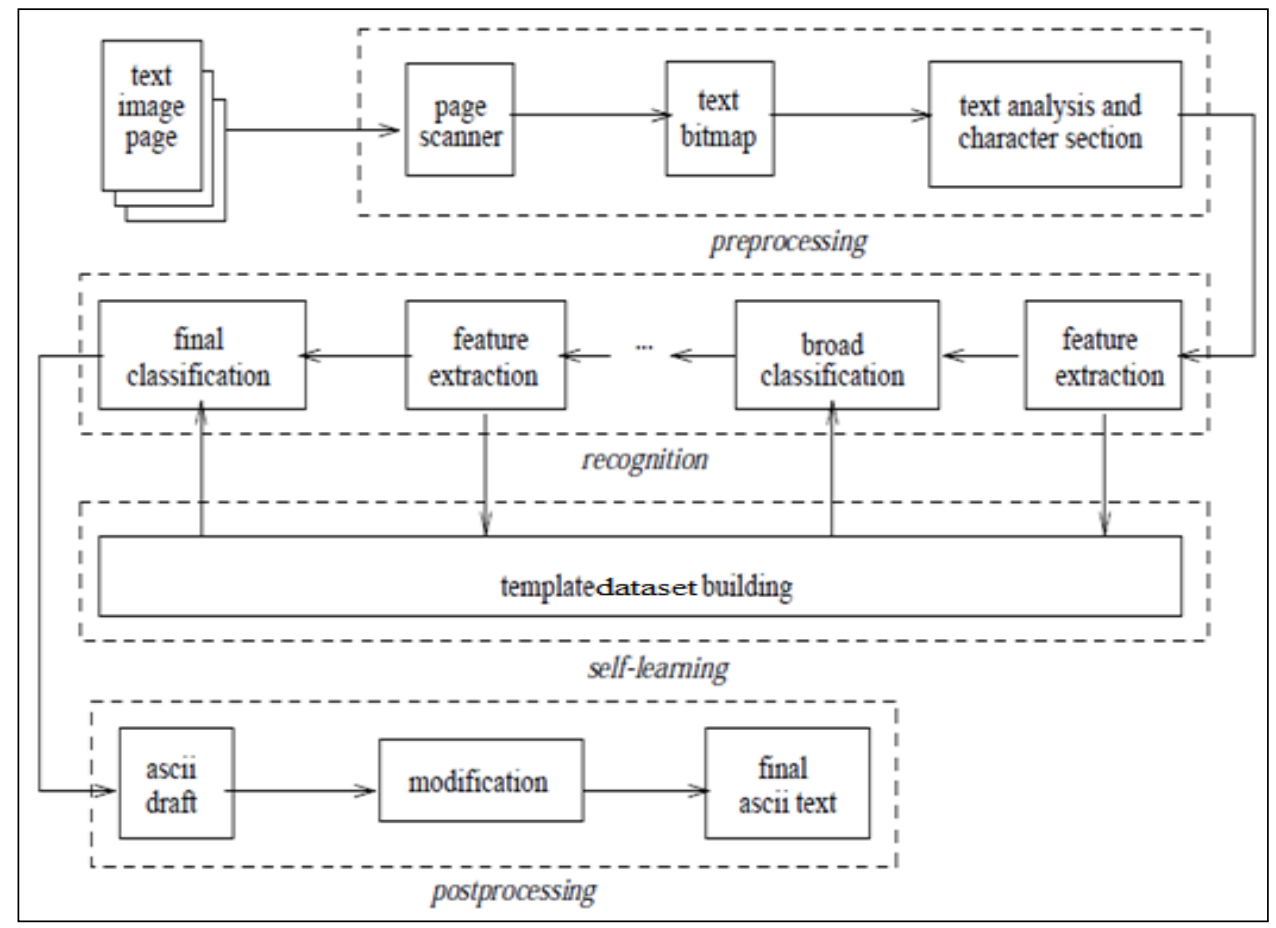

Figure 3. Architecture of the OCR system [27]

This research assume that the input image should be in RGB format. The input image will be converted into gray scale format. Then, the produced image will be converted into binary image using a binary threshold equation. The following procedure shows the steps of the proposed technique. Algorithm 1 is responsible for importing and filtering the input image. This step uses a page scanner to transfer the original text image into a bitmap file or a pixel matrix. Then, text analysis techniques will be performed to partition the image into lines and digits, and the position of each digit will be recorded in order to rebuild the image file.

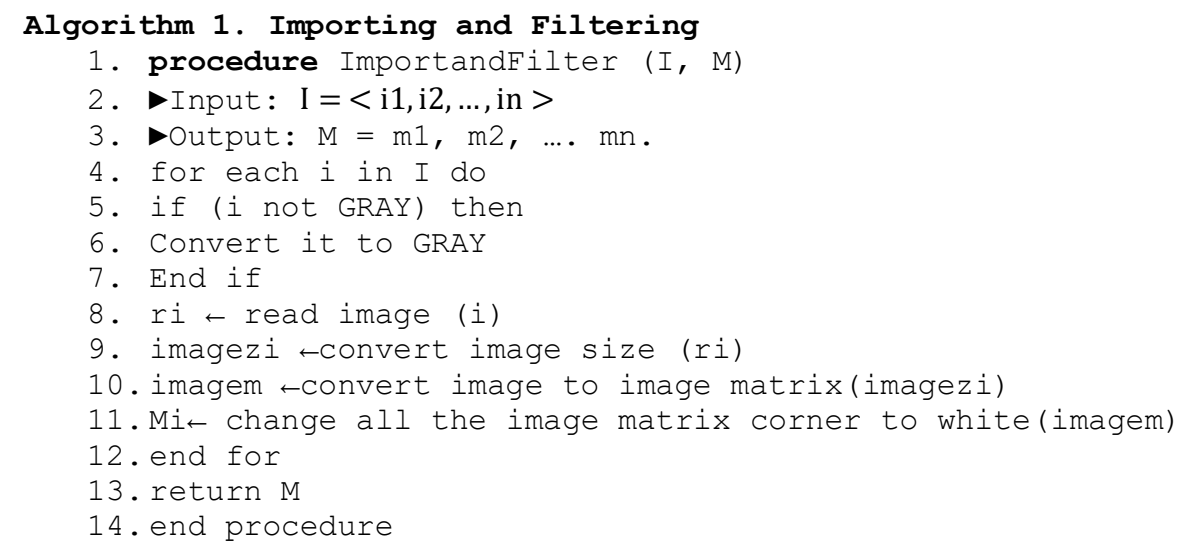


The above procedure considers $\mathrm{I}$ and $\mathrm{M}$ as the input and the output matrix representations, respectively. Number recognition is based on previously generated data set which contains the important features associated with the numbers which are recognized earlier. Algorithm 2 extracts the features of the input numbers and compares these features with those recorded in the data set used by the recognition process. If the features match or closely match, the input number will be classified into a class which contains all the numbers that have these common features.

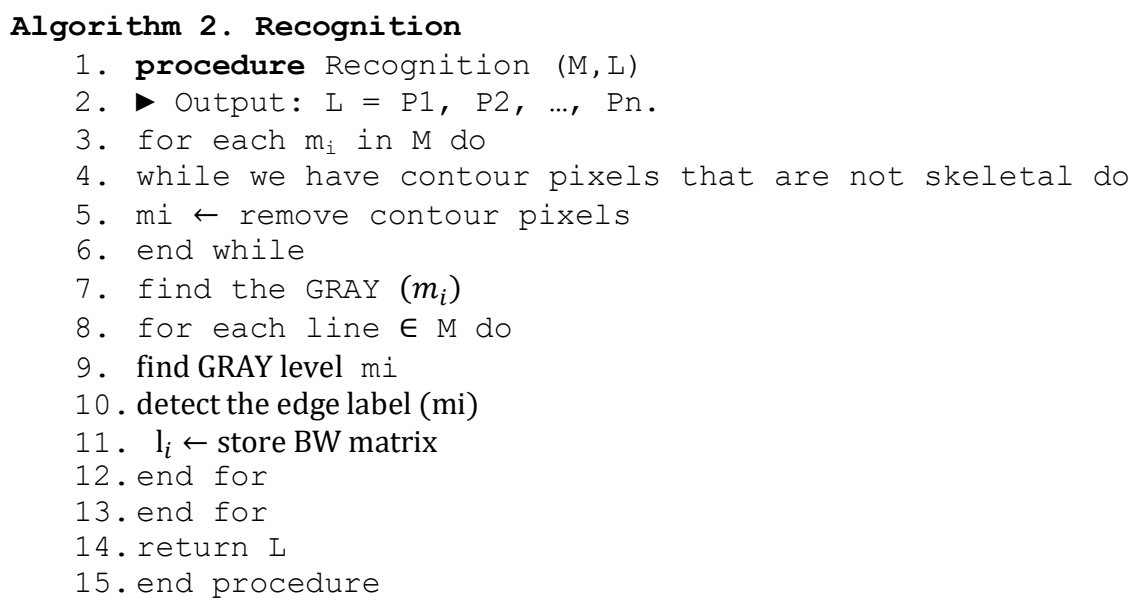

The purpose of this algorithm (lines 4-6) is to create the skeleton of a set of pixels by determining the skeletal pixels and the contour pixels, and then removing all the contour pixels that are not skeletal. This process will be repeated until a set which consists of only skeletal pixels is created. This set of templates will be used by a final classifier which is based on skeleton matching to find out which of them is the final result. In the final phase, Algorithm 3 compares the label with the data set to find the best matching number and saves it in a text file.

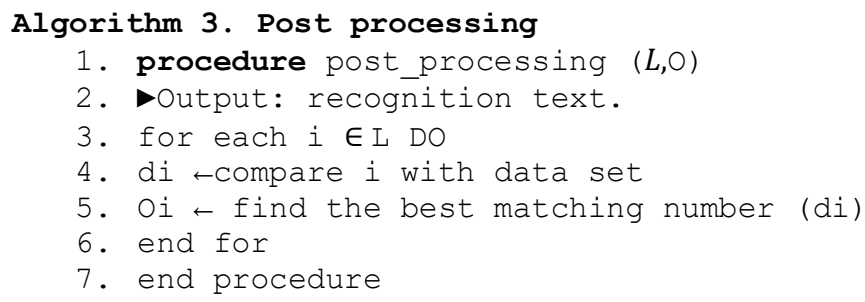

MATLAB has been used to implement the proposed system. MATLAB integrates mathematical computing and visualization and is considered a powerful software which provides a flexible environment for technical computing. According to the example shown in the Figure 4, when the user uploads image contains handwritten Arabic numbers, the system follows the previous process to display text file containing the recognized numbers as portrayed in Figure 5.

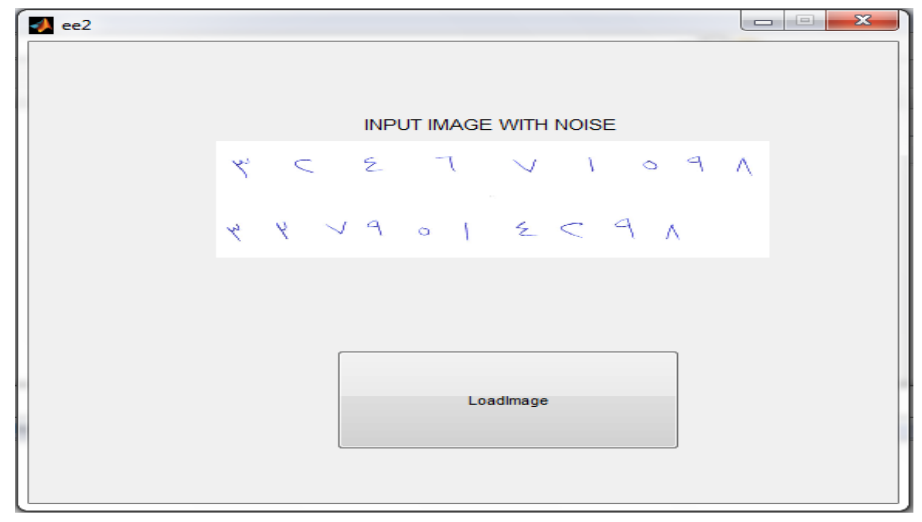

Figure 4. Sample of Arabic Handwritten numerals 


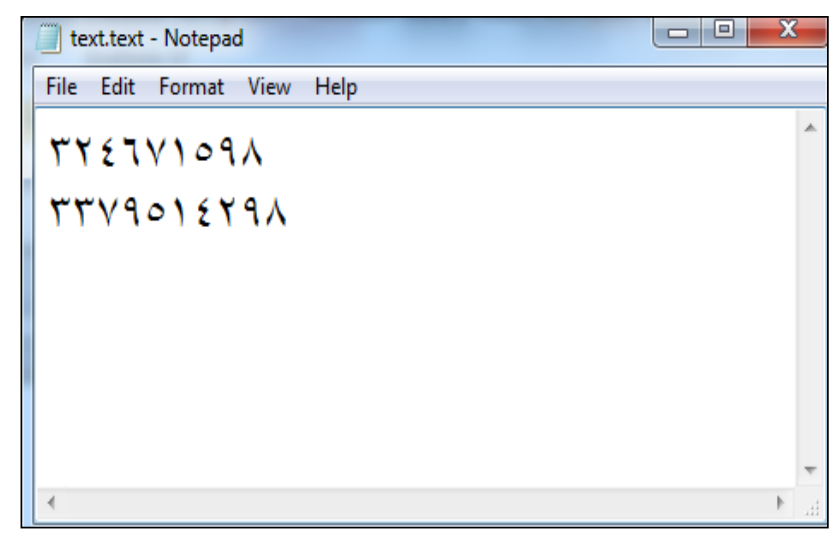

Figure 5. Text file contains the recognized numbers

\section{PERFORMANCE EVALUATION}

The data set that is being used in order to perform the experiments and to estimate the performance of the proposed system consists of 1000 different samples which contain multi-digit numbers. These samples were formed by collecting optically scanned samples of handwritten Arabic numerals. Each number consists of 1 to 20 digits in the range from 0 to 9 . These samples were recorded manually by 100 different persons of different age groups and sexes.

A page scanner is used to transfer the original text image into a pixel matrix. All these samples are converted to pixel images first and then converted to binary images. Text analysis techniques are applied next to partition the image into lines and digits. The position of each digit is recorded in order to rebuild the image file. Number recognition is based on previously generated data set which contains the important features related to the numbers which are known already. The recognition performance results on the test data sets for every single number are given in Table 2.

Table 2. Recognition results for each number

\begin{tabular}{cc}
\hline Arabic Number & Testing Accuracy of theNumber \\
\hline 0 & $99 \%$ \\
1 & $99 \%$ \\
2 & $98 \%$ \\
3 & $99 \%$ \\
5 & $100 \%$ \\
6 & $100 \%$ \\
7 & $99 \%$ \\
8 & $100 \%$ \\
9 & $100 \%$ \\
Average recognition & $99 \%$ \\
\hline
\end{tabular}

After analyzing and evaluating the results of our approach using the test samples and validation accuracy, it's obvious that this approach can easily detect the image features more accurately, making the accuracy 99.3 percent. This accuracy score exceeds the highest recorded accuracy for Arabic handwritten digit recognition.

\section{CONCLUSION AND FUTUER WORK}

In this study, an innovative technique based on OCR Skeleton matching method for automated recognition of Arabic handwritten numerals is presented. This approach involved image acquisition and filtering method, thinning algorithm design, segmentation technique, feature extraction and classification strategy. Also, the various methodologies used in OCR by recent research was reviewed in this research. This study aims to provide a useful guidance for new readers and researchers in the field of numeral recognition system. Experiments were performed to observe the system performance. A data set of 1000 multi-digit samples written by 100 persons is used in the experimentation. An average recognition rate of $99.3 \%$ were obtained.

In future, we aim to develop a system which combine other approaches like contour analysis to increase the recognition rate of numeral digits. Although our system can deal with overlapped numbers, we aim to treat joined and overlapped numbers more seriously and propose further enhancements to separate them. 


\section{REFERENCES}

[1] R. Al-Hmouz, "OCR based pixel fusion,” Journal of Applied Sciences, vol. 12, no. 22, pp. 2319-2325, 2012.

[2] M. H. Shirali-Shahreza, K. Faez, and A. Khotanzad, "Recognition of handwritten Persian/Arabic numerals by shadow coding and an edited probabilistic neural network," in Proceedings., International Conference on Image Processing, Washington, DC, USA, pp. 436-439, 1995.

[3] S. N. Nawaz, M. Sarfraz, A. Zidouri, and W. G. Al-Khatib, "An approach to offline Arabic character recognition using neural networks," in 10th IEEE International Conference on Electronics, Circuits and Systems, 2003. ICECS 2003. Proceedings of the 2003, Sharjah, pp. 1328-1331, 2003.

[4] S. Mozaffari, K. Faez, and H. R. Kanan, "Recognition of isolated handwritten Farsi/Arabic alphanumeric using fractal codes," in 6th IEEE Southwest Symposium on Image Analysis and Interpretation, 2004., Lake Tahoe, NV, USA, pp. 104-108, 2004.

[5] M. Takruri, R. Al-Hmouz, A. Al-Hmouz, and M. Momani, "Fuzzy c means based hybrid classifiers for offline recognition of handwritten indian (Arabic) numerals," International Journal of Applied Engineering Research, vol. 10, no. 1, pp. 1911-1924, 2015.

[6] H. Soltanzadeh, and M. Rahmati, "Recognition of Persian handwritten digits using image profiles of multiple orientations," Pattern Recognition Letters, vol. 25, no. 14, pp. 1569-1576, 2004.

[7] J. Sadri, C. Y. Suen, and T. D. Bui, "Application of support vector machines for recognition of handwritten Arabic/Persian digits," in Proceedings of Second Iranian Conference on Machine Vision and Image Processing, Tehran,Iran, pp. 300-307, 2003.

[8] S. A. Mahmoud, "Arabic (Indian) handwritten digits recognition using Gabor-based features," in 2008 International Conference on Innovations in Information Technology, Al Ain, pp. 683-687, 2008.

[9] S. M. Awaidah, and S. A. Mahmoud, "A multiple feature/resolution scheme to Arabic (Indian) numerals recognition using hidden Markov models,” Signal Processing, vol. 89, no. 6, pp. 1176-1184, 2009.

[10] M. Ziaratban, K. Faez, and F. Faradji, "Language-based feature extraction using template-matching in Farsi/Arabic handwritten numeral recognition," in Ninth International Conference on Document Analysis and Recognition (ICDAR 2007), Parana, pp. 297-301, 2007.

[11] M. O. Al barraq, and S. Mehrotra, "Recognition of Arabic handwritten amount in cheque through windowing approach," International Journal of Computer Applications, vol. 115, no. 10, 2015.

[12] M. Dehghan, and K. Faez, "Farsi handwritten character recognition with moment invariants," in Proceedings of 13th International Conference on Digital Signal Processing, Santorini, Greece, 1997, pp. 507-510.

[13] A. Al-Haj, "Combined DWT-DCT digital image watermarking," Journal of computer science, vol. 3, no. 9, pp. 740-746, 2007.

[14] A. Lawgali, "An Evaluation of Methods for Arabic Character Recognition," International Journal of Signal Processing, Image Processing and Pattern Recognition, vol. 7, no. 6, pp. 211-220, 2014.

[15] F. Al-Omari, "Handwritten Indian numeral recognition system using template matching approaches," in Proceedings ACS/IEEE International Conference on Computer Systems and Applications, pp. 83-88, 2001.

[16] I. Ahmad, S. A. Mahmoud, and G. A. Fink, "Open-vocabulary recognition of machine-printed Arabic text using hidden Markov models," Pattern Recognition, vol. 51, pp. 97-111, 2016.

[17] M. Takruri, R. Al-Hmouz, and A. Al-Hmouz, "A three-level classifier: fuzzy C means, support vector machine and unique pixels for Arabic handwritten digits," in 2014 World Symposium on Computer Applications \& Research (WSCAR), pp. 1-5, 2014.

[18] S. A. Mahmoud, and W. G. Al-Khatib, "Recognition of Arabic (Indian) bank check digits using log-gabor filters," Applied Intelligence, vol. 35, no. 3, pp. 445-456, 2011.

[19] J. Shi, and J. Malik, "Normalized cuts and image segmentation," IEEE transactions on pattern analysis and machine intelligence, vol. 22, no. 8, pp. 88-905, 2000.

[20] J. C. Bezdek, L. Hall, and L. P. Clarke, "Review of MR image segmentation techniques using pattern recognition," Medical physics, vol. 20, no. 4, pp. 1033-1048, 1993.

[21] A. Harifi, and A. Aghagolzadeh, "A new pattern for handwritten Persian/Arabic digit recognition," in Proc. of World Academy of Sci.,Eng. and Technology, pp. 130-133, 2005.

[22] M. H. Ghaleb, L. E. George, and F. G. Mohammed, "Printed and handwritten hindi/arabic numeral recognition using centralized moments," International Journal of Scientific \& Engineering Research, vol. 5, no. 3, pp. 140-144, 2014.

[23] A.-T. Ahmad, and H. Maen, "Recognition of on-line handwritten Arabic digits using structural features and transition network," Informatica, vol. 32, no. 3, pp. 2486-2495, 2008.

[24] N. Das, R. Sarkar, S. Basu, M. Kundu, M. Nasipuri and D. KumarBasu, "A genetic algorithm-based region sampling for selection of local features in handwritten digit recognition application, " Applied Soft Computing, vol. 12, no. 5, pp. 1592-1606, 2012.

[25] S. A. Mahmoud, I. Ahmad, W. G. Al-Khatib, M. Alshayeb, M. T. Parvez, V. Märgner, and G. A. Fink, "KHATT: An open Arabic offline handwritten text database," Pattern Recognition, vol. 47, no. 3, pp. 1096-1112, 2014.

[26] U. Saeed, "Automatic recognition of handwritten arabic text: A survey," Life Science Journal, vol. 11, no. 3s, pp. 232-235, 2014.

[27] N. Li, "An implementation of ocr system based on skeleton matching," Computing Laboratory, University of Kent, Canterbury, UK, 1993. 


\section{BIOGRAPHIES OF AUTHORS}
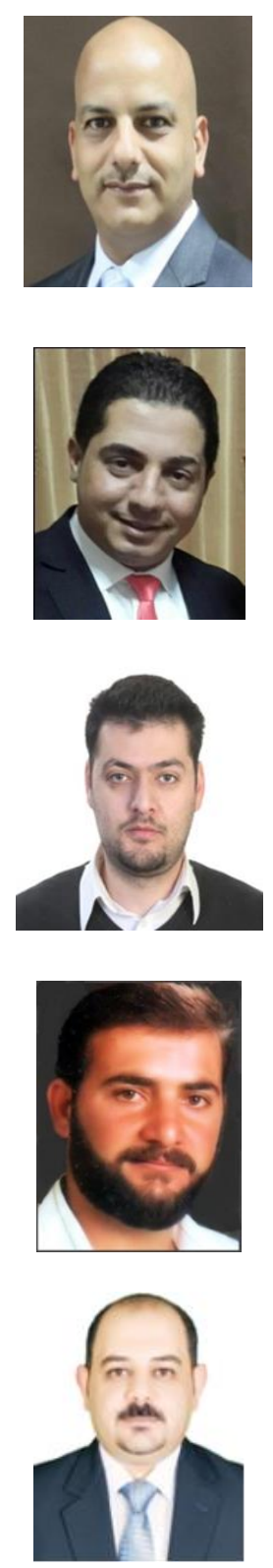

Bassam a. Y. Alqaralleh received his BSc degree in Computer Science in 1992, graduate Diploma in Computer Information Systems in 2002 and master's degree in computer science in 2004. After that, he received his PhD degree from University of Sydney in 2010. His research interests include Distributed Systems, Smart Technology, Innovative Technology Acceptance, Wireless Networking, Load-Balancing, mobile banking, mobile government and Security Systems. He has published several conference papers, journal papers and book chapters in these topics. Currently, He is an associate professor in Computer Science Department/Faculty of IT at Al-Hussein Bin Talal University (AHU)/Jordan.

Malek z. Alksasbeh received his B.S. degree in Computer science from Mu'tah University, Jordan, in 2005, and the M.S. and Ph.D. degrees in Information Technology from the University Utara Malaysia (UUM), Malaysia, in 2008 and 2012, respectively. Dr. Alksasbeh is Associate Professor in the College of Information Technology at Al-Hussein Bin Talal University (AHU). His research interests are in the areas of Technology Acceptance, Information Retrieval, Knowledge Managements, and Mobile Learning.

Tamer Abukhalil received the B.S. degree in computer science from Al-Albayt University, Mafraq, Jordan, in 2003 and the M.S. degree in computer science from Bangalore University, Bangalore, India, in 2007. In 2015 he obtained his Ph.D. degree in computer science and engineering at University of Bridgeport, Bridgeport, CT, USA. He is currently an assistant professor at AHU University, Ma'an, Jordan. While pursuing his $\mathrm{PhD}$, Tamer was a Research Assistant with the department of Engineering. His research interest includes the development multi-robotic deployment interfaces, Image processing, Artificial intelligence, Expert systems, Swarm robotic systems, and Object-Oriented Programming.

Harbi Al-Mahafzah is an assistant professor in the college of information technology at AlHussein Bin Talal University. He received his B.Sc. in Computer in 1998 from University of Mysore, Mysore, India. He finished his M.Sc. in Computer Science, in 2007 from University of Mysore, Mysore, India. Harbi pursed his Ph.D. in Biometric, from University of Mysore, Mysore, India 2013.Research interesting are: Multi-Biometric, Image Processing, Pattern Recognition

Tawfiq Ahmed Al Rawashdeh received his B.S. degree in Computer science from Al-Hussein Bin Talal University (AHU), Jordan, in 2005, and the M.S. degrees in Computer Science from Al-Balqa' Applied University, Jordan, in 2011. Al Rawashdeh is Lecturer in the College of Information Technology at Al-Hussein Bin Talal University (AHU). His research interests are in the areas of Cloud computing and algorithms. 